\title{
Special Issue, Phenology 2010 Conference, Dublin, Ireland
}

\author{
Alison Donnelly • Bridget F. O'Neill • Amelia Caffarra • \\ Mark D. Schwartz
}

Published online: 9 September 2011

(C) ISB 2011

Changes in the timing of phenological events may be one of the earliest observed responses to rapid global climate warming and therefore provides a robust mechanism by which to demonstrate the impact of rising temperature on our environment. This special issue resulted from an international conference on phenology entitled "Phenology 2010: climate change impacts and adaptation" (http://www.tcd.ie/Botany/ phenology/2010/), held in Trinity College Dublin, Ireland, in June 2010. The conference took a multidisciplinary approach to discuss advances in phenology research and brought together experts from around the world in the fields of plant and animal phenology from both terrestrial and aquatic ecosystems. There were four research themes; the first, an overarching theme on climate change (including modelling) with the remaining three underpinning themes on synchrony/ asynchrony, genetic adaptation, and biodiversity. The conference brought together 130 delegates from 24 countries around the world and featured 72 oral presentations and 42 poster presentations. "Phenology 2010" aimed to be the first in a series of biannual phenology conferences to be held at various locations. Dist. Professor Mark D. Schwartz, will host the next in the series during September 2012, at his home institution, the University of Wisconsin-Milwaukee, USA.

A. Donnelly $(\bowtie) \cdot$ B. F. O’Neill $\cdot$ A. Caffarra

Centre for the Environment, School of Natural Sciences,

Trinity College Dublin,

Dublin, Ireland

e-mail: Alison.Donnelly@tcd.ie

M. D. Schwartz

Department of Geography, University of Wisconsin-Milwaukee,

PO Box 413, Milwaukee, WI 53201, USA

A. Caffarra

Research and Innovation Centre, Agriculture area,

Fondazione Edmund Mach,

San Michele all'Adige,

Trento, Italy

A select number of papers were chosen, which underwent a rigorous peer-review process and resulted in the 14 manuscripts contained in this special issue. Given the broad range of subjects (climate change; forest phenology; pollen phenology; bird phenology; remote sensing; modeling; biodiversity; conservation; trophic interactions; phenotypic plasticity; genetic adaptation and citizen science) covered during the conference and the inability to publish all contributions we endeavored to select topics which reflected a number geographical regions (Australia; Mediterranean; Brazil; Scandanavia; North America and Europe) a variety of species (mainly plants and birds) and habitats (terrestrial and aquatic), and which would be of interest to a wide audience.

The contributions can be summarized as follows: Demarée and Rutishauser present an interesting debate on the origins of the word "phenology", which traces the term back to the midnineteenth century. The question of phenotypic versus genotypic control of leaf phenology in trees is explored by Charrier et al. and Friedman et al. in Europe and North America respectively. A number of studies of bird phenology examine (a) the influence of wind direction on migrating birds in Spain (Barriocanal et al.) and (b) the use of long-term datasets in Norway to estimate the timing of breeding and the ecological impact of climate change (Nilsson et al.). In order to examine synchrony/asynchrony between interdependent phenophases Donnelly et al. review examples from natural, agricultural and aquatic ecosystems. In Norway, Wielgolaski investigates the relationship between plant phenology and temperature over a 50-year period while Beaubien presents results from 20 years experience of a citizen science phenology network in Canada. Morellato et al. and Estiarte et al. examine reproductive phenology of trees along a transect from coast to inland in two very different habitats, a Brazilian forest and a Mediterranean scrub. Cornelius et al. compare methods to estimate seasonal phenological development from $\mathrm{BBCH}$ scale recording and Hudson et al. present a mapping technique to visualize synchrony between species. Jenouvrier 
and Visser suggest a new model for coupling population and phenological changes with global warming. Furthermore, Menzel et al. present advanced statistical techniques to examine extreme early and late phenological events.
Finally, the other guest editors would like to express our thanks to Dist. Professor Mark D. Schwartz who was instrumental in making this special issue of the International Journal of Biometeorology a reality. 